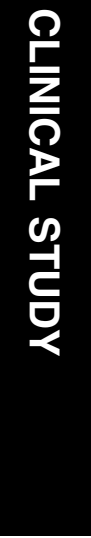

${ }^{1}$ The Vision Cooperative Research Centre, Sydney, Australia

${ }^{2}$ The Institute for Eye Research, Sydney, Australia

${ }^{3}$ Mansfield University of Pennsylvania, Pennsylvania, PA, USA

${ }^{4}$ School of Optometry and Vision Science, The University of New South Wales, Sydney, Australia

Correspondence:

P Lazon de la Jara, Vision Cooperative Research Centre,

PO Box 6327,

UNSW,

Sydney, NSW 1466,

Australia

Tel: + 61293857456 ;

Fax: +6129385 7401 .

E-mail:p.lazon@

ier.org.au

Received: 12 November 2008

Accepted in revised form: 17 April 2009

Published online: 15 May 2009

This study was supported in part by the Australian Federal Government through the Vision Cooperative Research Centre, Sydney, Australia.

Proprietary or Financial Interest: None

\title{
Pre-operative quality of life and psychological factors that influence patient decision making in LASIK
}

\section{Abstract}

Purpose To assess pre-operative visual function, psychological factors, personality traits and satisfaction in myopic patients seeking laser-assisted in situ keratomileusis (LASIK).

Methods Eligible patients seeking LASIK $(n=183)$ and successful contact lens wearers $(n=23)$ not interested in LASIK completed the Institute for Eye Research Quality of Life (QOL) Scale. Of the 183 patients seeking LASIK who completed the QOL instrument before LASIK, 30 declined the procedure and 153 underwent LASIK. One hundred and two patients who underwent LASIK attended their 3-month post-operative follow-up appointment and 51 patients failed to present for their post-operative follow-up appointment. Multiple comparisons (One-way Analysis of Variance (ANOVA)) were conducted to determine differences in visual function, psychological factors, personality traits and satisfaction between patient groups. Results Successful soft contact lens (SCL) wearers reported better functional vision $(P=0.001)$, felt more attractive $(P=0.007)$, had a lower frequency of disturbing visual and ocular symptoms $(P=0.027)$ and higher overall satisfaction with their current optical correction $(P<0.001)$ than patients seeking LASIK. LASIK patients lost to follow-up showed higher self-efficacy, adaptability and well-being than the patients who completed their appointments and those who declined surgery $(P<0.05)$.

Conclusions Psychological characteristics, the perception of visual and ocular symptoms and satisfaction with the current method of optical correction play an important role in the decision to undergo LASIK. The IER QOL
P Lazon de la Jara ${ }^{1,2}$, D Erickson 1,2,3, P Erickson ${ }^{2}$ and F Stapleton ${ }^{1,2,4}$
Scale is a valid and reliable instrument able to discriminate between different groups seeking choices for myopic correction. Psychological factors may influence the level of satisfaction with LASIK.

Eye (2010) 24, 270-275; doi:10.1038/eye.2009.115; published online 15 May 2009

Keywords: quality of life; psychological factors; LASIK

\section{Introduction}

Quality of Life (QOL) is a complex concept to define and to assess. It is a generic term that usually refers to expectations, goals, concerns, and degrees of satisfaction related to a person's life $\mathrm{e}^{1-4}$ and it is usually evaluated using self-assessment questionnaires.

Recently, the ophthalmic community has developed appropriate QOL instruments for the correction of refractive error with spectacles, contact lenses and refractive surgery. ${ }^{5-9}$ These instruments aim to assess the effect and correction of refractive errors on QOL. Several surveys indicate that laser-assisted in situ keratomileusis (LASIK) patients are highly satisfied with the outcome, ${ }^{10-13}$ despite the presence of visually disturbing phenomena such as haloes, glare and night vision problems. Most of these surveys focused on the measurement of visual function and satisfaction after LASIK. We assume that the assessment of QOL in refractive surgery should extend beyond these aspects and should include psychological factors and personality traits that may influence QOL. Further, comparisons of QOL have been made between emmetropes, refractive surgery patients, spectacles and contact lens wearers. ${ }^{14,15}$ These studies reported 
an improvement in QOL after refractive surgery, however it will be of interest to explore factors related to QOL in myopes seeking refractive surgery.

The Institute for Eye Research Multidimensional Quality of Life (IER QOL) Scale for Myopia focuses on vision problems related to myopia and has shown an ability to discriminate between different correction modalities. ${ }^{16}$ This scale was developed specifically for the assessment of functional dimensions related to the correction of myopia with spectacles, contact lenses and refractive surgery for pre-presbyopes plus the assessment of psychological variables and personality traits. This multidimensional scale addresses psychological variables, as well as vision and ocular problems that may arise because of the myopia treatment and influence patients' perceptions of health-related QOL.

This study was designed to explore differences in visual function, psychological factors, personality traits and satisfaction between myopic patients seeking LASIK and to determine whether these differences were associated with the patient's final choice of myopia correction using the IER QOL Scale.

\section{Materials and methods}

\section{Patients}

Myopic patients were recruited from the patient pool at the Centro de Cornea, Catarata y Glaucoma and Oftalmo Salud. Both are ophthalmic surgery practices in Lima, Peru, which provide a service to members of the general public seeking LASIK surgery. The investigation was conducted in accordance with the tenets of the Declaration of Helsinki. Approval by The University of New South Wales Human Research Ethics Committee was obtained and all patients were required to sign a declaration of Informed Consent.

One hundred and eighty-three consecutive myopic patients seeking LASIK $(112 \mathrm{~F}, 71 \mathrm{M})$ with a mean spherical equivalent of $-4.29 \mathrm{D} \pm 2.25$ and a mean age of $30 \pm 6$ years old were enroled. In addition, 23 (17F, $6 \mathrm{M})$ successful soft contact lens (SCL) wearers not seeking LASIK with a mean spherical equivalent of $-4.40 \mathrm{D} \pm 2.48$ and a mean age of $29 \pm 3$ years old were included for comparison.

Patients seeking LASIK presented wearing their habitual visual correction (spectacles and/or contact lenses) for their first LASIK screening appointment and were asked to complete the IER QOL Scale based on their perceived visual function with their habitual correction. Before completing the questionnaire, patients seeking LASIK were unaware of their eligibility for the surgical procedure. SCL wearers were asked to complete the IER
QOL Scale, while they were waiting for their follow-up contact lens appointments.

Of the patients seeking LASIK, three groups were identified as follows:

1. Patients who underwent LASIK and who completed their 3-month follow-up appointment (Completed group, $n=102$ ).

2. Patients who underwent LASIK but did not attend their 3-month follow-up appointment (Lost to follow-up group, $n=51$ ).

3. Patients who made a personal decision to decline LASIK, despite fulfilling the inclusion criteria (Declined group, $n=30$ ).

We certified that all applicable and governmental regulations concerning the ethical use of human volunteers were followed during this research.

\section{Assessment tool}

The IER QOL scale

The IER QOL Scale assesses physical status, psychological state, personality traits and cosmesis, which are dimensions associated with a patient's QOL. In addition, the scale assesses patient satisfaction with the current treatment for correcting myopia.

Physical status relates to visual and ocular symptoms that a patient experiences with the corrective method of myopia and tolerance to these symptoms. Psychological state refers to how a patient adjusts to different situations and includes subjective well-being, self-efficacy and adaptability. ${ }^{17}$ Subjective well-being describes how optimistic or pessimistic a person feels when facing a particular situation. ${ }^{18,19}$ Self-efficacy is, how much a person believes that, he/she can succeed in a given specific treatment or situation. ${ }^{19}$ Adaptability describes the ability to adapt or adjust to different conditions, such as a new environment, or physical status. ${ }^{18-20}$ Conversely, personality traits such as extraversion or introversion refer to a person's behaviour. Extraversion and introversion affect how a patient perceives and reports symptoms to healthcare professionals. ${ }^{21-23}$ Cosmesis evaluates the person's view of how the correction affects his/her attractiveness. ${ }^{24}$

The IER QOL Scale has the following subscales:

- Frequency of disturbing visual and ocular symptoms

- Tolerance to disturbing visual and ocular symptoms

- Health Proneness Psychological Traits Questionnaire $(\mathrm{HPQ})^{25}$

- Personality traits (extraversion/introversion)

- Cosmesis

This multidimensional scale comprises 26

vision-oriented items (13 items related to frequency 
of disturbing visual and ocular symptoms, 13 items corresponding to tolerance of visual and ocular symptoms), 13 psychologically and personality trait-oriented items (health proneness questionnaire (HPQ) which includes 10 items related to adaptability, self-efficacy and subjective well-being and three personality trait items related to extraversion/ introversion) and three items measuring cosmesis. In addition, there are five questions related to satisfaction. A higher score for the tolerance and frequency of disturbing visual and ocular symptoms subscales indicated poorer performance. Higher scores for the HPQ subscale mean patients are more likely to be satisfied with life, adapt to new treatments, have adequate coping strategies and verbalize symptoms. The cosmesis subscale means appearance was important.

\section{Translation and internal consistency of the Spanish IER QOL scale}

The IER QOL Scale was validated earlier by Erickson and co-workers ${ }^{16}$ and they reported acceptable overall construct validity and reliability.

The IER QOL Scale was translated into Spanish for this particular study. Two translators (native Spanish speakers) translated the English version into Spanish. The translators were briefed that, emphasis should be placed on conceptual rather than literal translation; so that translated questions convey equivalent meanings. Translators met and compared both translations and, at the end, they agreed on a common forward Spanish translation.

The forward translation and the original English version were given to another translator, a British English native speaker. The English original version and back-translated Spanish versions were compared to find the best fit. The quality of the translation was rated and, if a translated item showed some inconsistency, an alternative translation was proposed. Only minor inconsistencies were found and the alternative translation was discussed with the researcher in charge of this study. After this process, a final Spanish version was produced.

In a preliminary study, the Spanish IER QOL Scale was administered once to 115 myopic patients seeking refractive surgery from one of the ophthalmic centres involved in the study to determine the internal consistency with the Cronbach's $\alpha$ coefficient. When the Spanish IER QOL Scale was completed, patients did not know at that time if they were suitable candidates for refractive surgery. Cronbach's $\alpha$ coefficients for the subscales of the IER QOL Scale Spanish version were 0.77 on the cosmesis factor, 0.80 on the HPQ, 0.73 on the extraversion/introversion factor, 0.88 on the tolerance factor, 0.88 on the frequency factor and 0.84 for the entire scale. This shows a strong internal consistency and these results are similar to the ones encountered with the original English version. No redundancy on the items of the subscales was found.

Data from the validation study were used to estimate the required sample size for the main study, namely that the grouped standard deviation across subscales was 0.8 units and 0.75 units represented a significant change between groups. A minimum sample size of 30 patients per subgroup was established based on estimates of type I error $\alpha=0.05$, for a power of $90 \%$, assuming a null hypothesis that there were no differences in pre-operative sub-groups.

\section{Statistical analysis}

Pre-operative QOL dimensions of the IER QOL Scale were compared between genders in the group of patients seeking LASIK.

Repeated measures of one-way Analysis of Variance (ANOVA) was used to test for differences in pre-operative QOL dimensions between the completed group, lost to follow-up group, declined group and SCL wearers group. A multiple comparisons test with Bonferroni adjustment was used to determine differences between QOL dimensions before LASIK. Statistical significance was set at $P<0.05$.

SPSS for Windows 11.5 (SPSS, Inc, Chicago, IL, USA) software was used for the analysis.

\section{Results}

Pre-operative IER QOL scale differences in gender between patients seeking LASIK

The IER QOL Scale detected significant differences between men $(n=71)$ and women $(n=112)$ seeking LASIK in cosmesis $(P=0.050)$ and tolerance of visual and ocular symptoms $(P=0.029)$. Women disliked their appearance more with spectacles in social and professional situations and were able to tolerate more visual and ocular symptoms than men. No significant differences were found in the other QOL dimensions assessed pre-operatively between men and women.

\section{Pre-operative QOL dimensions between SCL wearers, completed, lost to follow-up and declined patient groups}

The one-way ANOVA detected significant differences between the four patient groups (SCL wearers group, completed group, lost to follow-up group, and the declined group) in the frequency of disturbing visual 
Table 1 One-way ANOVA pre-operative IER QOL scale dimensions across SCL wearers and subjects seeking LASIK

\begin{tabular}{|c|c|c|c|c|c|}
\hline $\begin{array}{l}\text { IER scale } \\
\text { dimensions }\end{array}$ & $\begin{array}{l}\text { Sum of } \\
\text { squares }\end{array}$ & $d f$ & $\begin{array}{l}\text { Mean } \\
\text { square }\end{array}$ & $\mathrm{F}$ & P-value \\
\hline \multicolumn{6}{|c|}{ Pre-operative satisfaction } \\
\hline Between groups & 267.189 & 3 & 89.063 & 17.007 & 0.000 \\
\hline Within groups & 1057.859 & 202 & 5.237 & & \\
\hline Total & 1325.049 & 205 & & & \\
\hline \multicolumn{6}{|l|}{ Pre-operative cosmesis } \\
\hline Between groups & 23.976 & 3 & 7.992 & .933 & 0.029 \\
\hline Within groups & 524.704 & 202 & 2.598 & & \\
\hline Total & 548.680 & 205 & & & \\
\hline \multicolumn{6}{|l|}{ Pre-operative $H P Q$} \\
\hline Between groups & 161.551 & 3 & 53.850 & 3.834 & 0.011 \\
\hline Within groups & 2837.265 & 202 & 14.046 & & \\
\hline Total & 2998.816 & 205 & & & \\
\hline \multicolumn{6}{|c|}{ Pre-operative extraversion } \\
\hline Between groups & 20.937 & 3 & 6.979 & 2.086 & 0.103 \\
\hline Within groups & 675.670 & 202 & 3.345 & & \\
\hline Total & 696.607 & 205 & & & \\
\hline \multicolumn{6}{|l|}{ Pre-operative tolerance } \\
\hline Between Groups & 12.385 & 3 & 4.128 & .128 & 0.943 \\
\hline Within Groups & 6510.916 & 202 & 32.232 & & \\
\hline Total & 6523.301 & 205 & & & \\
\hline \multicolumn{6}{|l|}{ Pre-operative frequency } \\
\hline Between Groups & 663.944 & 3 & 221.315 & 3.107 & 0.028 \\
\hline Within Groups & 14386.813 & 202 & 71.222 & & \\
\hline Total & 15050.757 & 205 & & & \\
\hline
\end{tabular}

$P$-values in bold are significant at the 0.05 level.

and ocular symptoms, HPQ, cosmesis and satisfaction subscales of the IER QOL Scale (Table 1).

Multiple comparisons testing showed that SCL wearers had a significantly lower frequency of visual and ocular symptoms $(20.5 \pm 6.2, P=0.023)$ than patients seeking LASIK from the lost to follow-up surgical group (26.6 $\pm 9.8, n: 51)$. Furthermore, SCL wearers reported a significantly higher score for cosmesis $(9.52 \pm 1.23$, $P=0.023)$ than the completed group $(8.43 \pm 1.7, n=102)$. SCL wearers felt more attractive in social and professional situations compared with those seeking LASIK. In addition, the SCL wearers reported a significantly higher overall satisfaction $(10.9 \pm 2.6$, $P<0.001)$ with their current optical correction in comparison with patients seeking LASIK from the completed group $(14.4 \pm 2.1, n=102)$, the lost to follow-up group $(14.7 \pm 2.4, n=51)$ and the declined group $(14.1 \pm 2.3, n=30)$. Lower scores for the satisfaction subscale indicate higher satisfaction. The lost to follow-up group showed a higher score on the HPQ subscale $(33.6 \pm 3.5, n=51)$ that was significantly different from the completed group $(31.6 \pm 3.9, n=102$,
Table 2 One-way ANOVA for HPQ subscale dimensions between patients seeking LASIK

\begin{tabular}{lrrrrr}
\hline$H P Q$ dimensions & Sum of squares & df & Mean square & $\mathrm{F}$ & P-value \\
\hline Adaptability & & & & & \\
$\quad$ Between groups & 38.158 & 2 & 19.079 & 4.414 & $\mathbf{0 . 0 1 3}$ \\
Within groups & 778.104 & 180 & 4.323 & & \\
$\quad$ Total & 816.262 & 182 & & & \\
& & & & & \\
Self-efficacy & & & & & \\
$\quad$ Between groups & 29.616 & 2 & 14.808 & 8.058 & $\mathbf{0 . 0 0 0}$ \\
Within groups & 330.788 & 180 & 1.838 & & \\
$\quad$ Total & 360.404 & 182 & & & \\
& & & & & \\
Well-being & & & & & \\
$\quad$ Between groups & 4.118 & 2 & 2.059 & 1.045 & 0.354 \\
Within groups & 354.778 & 180 & 1.971 & & \\
$\quad$ Total & 358.896 & 182 & & & \\
\hline
\end{tabular}

$P$-values in bold are significant at the 0.05 level.

$P=0.013)$, and the declined group $(31.3 \pm 3.4, n=30$, $P=0.046$ ), indicating a higher degree of self-efficacy, adaptability and subjective well-being in those patients who failed to return for follow up.

The HPQ was divided into its dimensions of adaptability, self-efficacy and subjective well-being. Significant differences were found between the finished surgical group, the lost to follow-up group and the declined group in adaptability and self-efficacy (Table 2).

Multiple comparisons testing showed that the lost to follow-up surgical group had a significantly higher adaptability score $(13.2 \pm 2.1, n=51, P=0.014)$ than the declined group $(11.8 \pm 1.8, n=30)$, and a higher self-efficacy score $(10.4 \pm 1.2, P<0.001)$ than the finished surgical group $(9.5 \pm 1.3, n=102)$.

\section{Discussion}

High myopia has been shown to be associated with some psychological effects such as lack of self-confidence, social isolation and difficulties forming relationships. ${ }^{26}$ These effects were augmented by cosmetic factors, such as wearing thick spectacle lenses. On the basis of these findings, we were interested in establishing whether cosmesis influenced the final decision for undergoing LASIK in this low to moderate myopic group. The IER QOL Scale detected that SCL wearers felt more attractive than patients seeking LASIK in social contexts. Moreover, patients in our study seeking refractive surgery had a higher frequency of disturbing visual and ocular symptoms and were less satisfied with their habitual optical correction, compared with a group of regular SCL wearers not interested in LASIK. Our findings suggest that attractiveness, a better visual function and higher satisfaction with current optical correction, as reported by the SCL wearers group, may be some of the 
factors that influence a patient's decision not to consider the option of a surgical procedure. This finding is consistent with an earlier report, ${ }^{27}$ where contact lens wearers who were coping well were easily discouraged from having laser surgery. Rankin et $a l^{28}$ in a study of patients who underwent elective cosmetic surgery, reported that dissatisfaction with appearance was the main reason for undergoing rhytidectomy (facelift), liposuction and rhinoplasty. We cannot make a general statement that perceptions in these types of patients were similar to the ones seeking LASIK; however, we found that the cosmesis data indicated that patients seeking LASIK did not feel attractive in social and professional situations. This result is consistent with an earlier study where pre-operative myopes disliked their appearance and were less satisfied with their current correction before surgery. ${ }^{29}$ Moreover, the IER QOL Scale detected that women disliked their own appearance more with spectacles and felt less attractive than did the men who wore spectacles. Self-perception of their attractiveness may be an important driver for patients seeking LASIK.

The IER QOL Scale identified that patients who underwent surgery were more likely to be the risk takers and to adapt more easily to new situations than the group of patients who declined LASIK. This is consistent with earlier findings. ${ }^{20}$ The IER QOL scale identified that these patients tended to be more spontaneous and more comfortable in new situations. This may imply that patients are prepared to accept the possibility of some level of visual compromise after surgery and being adaptable, prepared to accept this compromise for the trade-off of freedom from spectacles and contact lenses. We suspect that willingness to accept some visual compromise influences the high level of overall satisfaction reported earlier with refractive surgery. It seems that the patients who declined LASIK were not willing to take such risks, as they recorded lower adaptability scores than the patients who underwent LASIK.

Another interesting result is that the patients lost to follow-up, in comparison to the ones who completed their follow-up appointments up to the third month and the ones who declined LASIK, had a higher pre-operative HPQ score. When the subscale was divided into its dimensions (adaptability, self-efficacy and subjective well-being), adaptability and self-efficacy were statistically significantly different between these groups. Our interpretation is that patients lost to follow-up can adapt easily to new situations with a high sense of mastery of their environment (self-efficacy) and did not feel a need to complete the study.

Quality of life instruments can identify potential psycho-social problems that are not detected by clinical examination and this valuable information can be used to address issues that need to be resolved to improve the condition or treatment. Moreover, it can give further insight into understanding the potential effect of the treatment on patients' daily lives. However, limitations in the use of QOL instruments are related to how the individual interprets the consequences of his/her health condition. This interpretation is influenced by the individual's perceptions and social environment with consequent affects on QOL measures. Clearly, QOL reflects the health status from the patient's perspective.

We can hypothesize that psychological factors, the desire to improve visual function and perceived appearance, attractiveness, play important roles in the decision to undergo refractive surgery in those individuals who otherwise fulfill the inclusion criteria for surgery. Patients who decided to have LASIK showed a psychological profile of adaptability and risk taking. They were less concerned with possible visual problems and complications after surgery. Furthermore, the differences in psychological factors between LASIK patients are of interest. Lost to follow-up patients can adapt easily to new situations, because of their increased self-efficacy, adaptability and sense of subjective well-being. In contrast, patients who declined to have LASIK but were otherwise eligible, were likely to be more concerned about the consequences of LASIK in their QOL and vision, and were not willing to take such a risk.

We show in this study, that the IER QOL Scale is a highly valid and reliable multidimensional QOL screening instrument. It effectively differentiates visual function, psychological factors, cosmesis and satisfaction between SCL wearers not seeking LASIK and myopes seeking LASIK. Moreover, these dimensions were associated with the patient's final choice of myopia correction.

\section{Acknowledgements}

The authors thank refractive surgeons, Dr Miguel Asmat from Centro de Cornea, Catarata y Glaucoma, Lima, Peru and Dr Luis Izquierdo from Oftalmo Salud Insituto de Ojos, Lima, Peru for their unconditional support in conducting this study at their Eye Clinics and also Dr Judith Flanagan for her review and feedback on the final stages of this manuscript.

\section{References}

1 Muldoon MF, Barger SD, Flory JD, Manuck SB. What are quality of life measurements measuring? BMJ 1998; 316(7130): 542-545. 
2 Haas BK. Clarification and integration of similar quality of life concepts. Image J Nurs Sch 1999; 31(3): 215-220.

3 Morales AJ, Kettel LM. Quality of life assessment. Semin Reprod Endocrinol 1996; 14(2): 155-159.

4 Guyatt G, Feeny D, Patrick D. Issues in quality-of-life measurement in clinical trials. Control Clin Trials 1991; 12(4 Suppl): 81S-90S.

5 Vitale S, Schein OD, Meinert CL, Steinberg EP. The refractive status and vision profile: a questionnaire to measure vision-related quality of life in persons with refractive error. Ophthalmology 2000; 107(8): 1529-1539.

6 Brunette I, Gresset J, Boivin JF, Boisjoly H, Makni H. Functional outcome and satisfaction after photorefractive keratectomy. Part 1: development and validation of a survey questionnaire. Ophthalmology 2000; 107(9): 1783-1789.

7 Brunette I, Gresset J, Boivin JF, Pop M, Thompson P, Lafond GP et al. Functional outcome and satisfaction after photorefractive keratectomy. Part 2: survey of 690 patients. Ophthalmology 2000; 107(9): 1790-1796.

8 Berry S, Mangione CM, Lindblad AS, McDonnell PJ. Development of the National Eye Institute refractive error correction quality of life questionnaire: focus groups. Ophthalmology 2003; 110(12): 2285-2291.

9 Pesudovs K, Garamendi E, Elliott DB. The Quality of Life Impact of Refractive Correction (QIRC) Questionnaire: development and validation. Optom Vis Sci 2004; 81(10): 769-777.

10 McGhee CN, Craig JP, Sachdev N, Weed KH, Brown AD. Functional, psychological, and satisfaction outcomes of laser in situ keratomileusis for high myopia. J Cataract Refract Surg 2000; 26(4): 497-509.

11 McDonald MB, Carr JD, Frantz JM, Kozarsky AM, Maguen E, Nesburn AB et al. Laser in situ keratomileusis for myopia up to -11 diopters with up to -5 diopters of astigmatism with the summit autonomous LADARVision excimer laser system. Ophthalmology 2001; 108(2): 309-316.

12 Balazsi G, Mullie M, Lasswell L, Lee PA, Duh YJ. Laser in situ keratomileusis with a scanning excimer laser for the correction of low to moderate myopia with and without astigmatism. J Cataract Refract Surg 2001; 27(12): 1942-1951.

13 Miller AE, McCulley JP, Bowman RW, Cavanagh HD, Wang $\mathrm{XH}$. Patient satisfaction after LASIK for myopia. Clao J 2001; 27(2): 84-88.

14 Pesudovs K, Garamendi E, Elliott DB. A quality of life comparison of people wearing spectacles or contact lenses or having undergone refractive surgery. J Refract Surg 2006; 22(1): 19-27.

15 Chen CY, Keeffe JE, Garoufalis P, Islam FM, Dirani M, Couper TA et al. Vision-related quality of life comparison for emmetropes, myopes after refractive surgery, and myopes wearing spectacles or contact lenses. J Refract Surg 2007; 23(8): 752-759.
16 Erickson DB, Stapleton F, Erickson P, du Toit R, Giannakopoulos E, Holden B. Development and validation of a multidimensional quality-of-life scale for myopia. Optom Vis Sci 2004; 81(2): 70-81.

17 Diener E. Traits can be powerful but are not enough: lessons from subjective well-being. J Res Personality 1996; 30: 389-399.

18 Diener E. Subjective well-being. The science of happiness and a proposal for a national index. Am Psychol 2000; 55(1): 34-43.

19 Bernard LC, Hutchinson S, Lavin A, Pennington P. Ego-strength, hardiness, self-esteem, self-efficacy, optimism and maladjustment: health related personality constructs and the 'Big Five' model of personality. Assessment 1996; 3(2): 115-131.

20 Erickson DB, Ryan RA, Erickson P, Aquavella JV. Cognitive styles and personality characteristics strongly influence the decision to have photorefractive keratectomy. J Refract Surg 1995; 11(4): 267-274; discussion 274-281.

21 Feldman PJ, Cohen S, Doyle WJ, Skoner DP, Gwaltney Jr JM. The impact of personality on the reporting of unfounded symptoms and illness. J Pers Soc Psychol 1999; 77(2): 370-378.

22 Wade JB, Dougherty LM, Hart RP, Rafii A, Price DD. A canonical correlation analysis of the influence of neuroticism and extraversion on chronic pain, suffering, and pain behavior. Pain 1992; 51(1): 67-73.

23 Harkins SW, Price DD, Braith J. Effects of extraversion and neuroticism on experimental pain, clinical pain, and illness behavior. Pain 1989; 36(2): 209-218.

24 Erickson DB, Stapleton F, Erickson P, duToit R, Giannakopoulos E, Holden B. Development and validation of a multidimensional quality-of-life scale for myopia. Optom Vis Sci 2004; 81: 70-81.

25 Erickson D, Stapleton F, Erickson P, Giannakopoulous E, Wilson C. The development and validation of the health proneness questionnaire. J Clin Psych Med S 2006; 13: 411-419.

26 Rose K, Harper R, Tromans C, Waterman C, Goldberg D, Haggerty C et al. Quality of life in myopia. Br J Ophthalmol 2000; 84(9): 1031-1034.

27 Khan-Lim D, Craig JP, McGhee CN. Defining the content of patient questionnaires: reasons for seeking laser in situ keratomileusis for myopia. J Cataract Refract Surg 2002; 28(5): 788-794.

28 Rankin M, Borah GL, Perry AW, Wey PD. Quality-of-life outcomes after cosmetic surgery. Plast Reconstr Surg 1998; 102(6): 2139-2145; discussion 2146-2147.

29 Nichols JJ, Twa MD, Mitchell GL. Sensitivity of the National Eye Institute Refractive Error Quality of Life instrument to refractive surgery outcomes. J Cataract Refract Surg 2005; 31(12): 2313-2318. 